\title{
Pregnancy in a bicornuate uterus with contraceptive coil in situ - a case report
}

\begin{abstract}
Methods of contraception are being designed to become increasingly reliable however, patients still get pregnant after a contraceptive fails. Probably the most dramatic of these situations is when a patient has an intrauterine device and still manages to conceive. This is precisely what happened in this case. The patient attended the emergency room to confirm her pregnancy and on transabdominal and transvaginal scan both a viable pregnancy and a coil were demonstrated. It appeared that the uterus was bicornuate which makes for more interesting images and which may have contributed to the failure of the contraceptive method in question.
\end{abstract}

Keywords: Bicornuate uterus, Congenital uterine anomalies, Mullerian duct anomalies, Intrauterine contraceptive device, Contraceptive device failure, Ultrasonography of intrauterine devices
Volume 7 Issue 3 - 2017

\author{
Olivia Anne Cassar, Silvaine Marie Dalli \\ Mater Dei Hospital, University of Malta, Malta
}

Correspondence: Olivia Anne Cassar, Mater Dei Hospital, University of Malta, Malta, Email oliviaannesammut@gmail.com

Received: June 06, 2017| Published: June 16, 2017

\section{Introduction}

When choosing a contraceptive more and more women are opting for Long Acting Reversible Contraception or LARCs as these methods do not depend on the patient herself to take them or use them to be effective. ${ }^{1}$ There are several such LARCs but one of the most common is the intrauterine device (IUD) as it is non hormonal and inexpensive. It is quoted as being over $99 \%$ effective which means that less than 2 women in 100 will get pregnant over 5 years. ${ }^{2}$ The copper at its core acts by having a toxic effect on the sperm and ova and decreasing sperm motility. It also impedes implantation. Different coils are licensed for different lengths of time ranging from 5 to 10 years.

In the case in question, the patient was incidentally also found to have a bicornuate uterus. This is a congenital uterine anomaly also called a Mullerian duct anomaly. These are congenital anomalies that occur when the Mullerian ducts or paramesonephric ducts do not develop correctly in the embryonic period. They are quite common but the majority is asymptomatic. ${ }^{3}$ There are several types and the main modes of diagnosis are ultrasound and MRI. Bicornuate uteri are thought to represent $25 \%$ of all Mullerian duct anomalies ${ }^{4,5}$ and occur when there is a partial failure of fusion of the ducts, resulting in a uterus divided into two horns. In most cases, a bicornuate uterus is incidentally discovered when the pelvis is imaged like in this case. It can also be picked up during the investigation of a patient for sub fertility or recurrent miscarriage.

\section{Case}

In the case presented the patient, a 28-year old student studying English in Malta presented to the Gynaecology emergency room demanding to have her pregnancy confirmed. This was deemed to be an inappropriate referral but on closer questioning the patient admitted to a positive pregnancy test and also to having had an intrauterine device inserted a few months previously.

In view of this, she had not suspected pregnancy straight away and had not had a scan yet. It was thought her anxiety was therefore justified and thus an ultrasound was performed to confirm the pregnancy and its location. This is because studies have shown that an ectopic pregnancy is more likely if a patient is using an intrauterine device. ${ }^{6}$ She gave her verbal consent readily and an abdominal scan was performed in the emergency room. The patient denied having a full bladder which is usually a prerequisite to performing an abdominal ultrasound however she had a low BMI so the plan was to start abdominally first and then proceed to a trans-vaginal scan if this was deemed necessary.

On placing the curvilinear probe on the patient's abdomen a sagittal view of the uterus was obtained. The bladder was only slightly filled however a good view of the uterus, cervix, and vagina was obtained. Usually, in this view, the endometrial stripe is apparent. In this case, it became apparent that the uterus had a congenital anomaly as 2 horns were clearly visible (Figure 1).

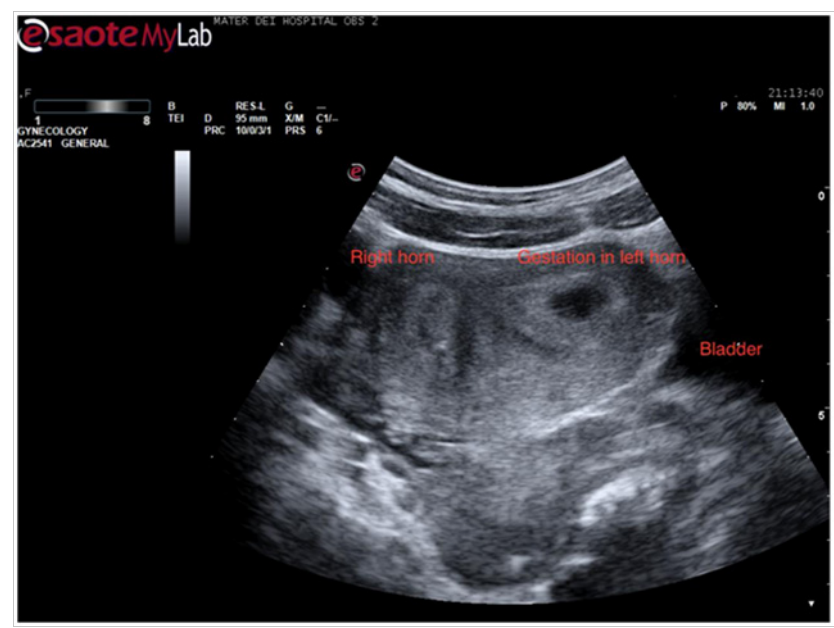

Figure I Trans abdominal ultrasound showing a sagittal view of the uterus. Two horns are apparent with a possible gestational sac in the left horn.

A closer look at the hypo echoic mass in the right horn of the uterus revealed what appeared to be a gestational sac measuring almost $20 \mathrm{~mm}$. By zooming in onto the sac a definite fetal pole together with a yolk sac came into view. This confirmed the hypo echoic area to be a gestation sac and hence an intrauterine pregnancy (Figure 2). The pregnancy was viable as a fetal heart beat was seen. 


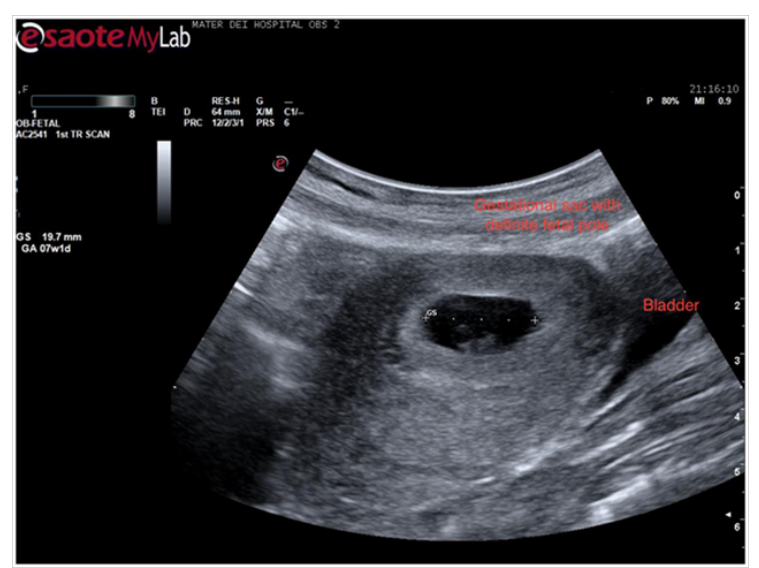

Figure 2 Transabdominal scan showing a transverse view of the uterus with a definite gestational sac inside one of the horns.

Returning to the sagittal view of the uterus the entire uterus was visualized and on sliding the probe across the uterus in this plane, the intrauterine contraceptive device came clearly into view (Figure 3). This was quite a dramatic view as the whole intrauterine device was seen to be placed inside the body of the uterus. The patient became visibly anxious at this stage so care was taken to reassure her and explain the findings thus far. By sliding the probe again an image was obtained showing both the coil and the gestation sac in the same view which confirmed that this was a case of pregnancy with a coil in situ (Figure 4).

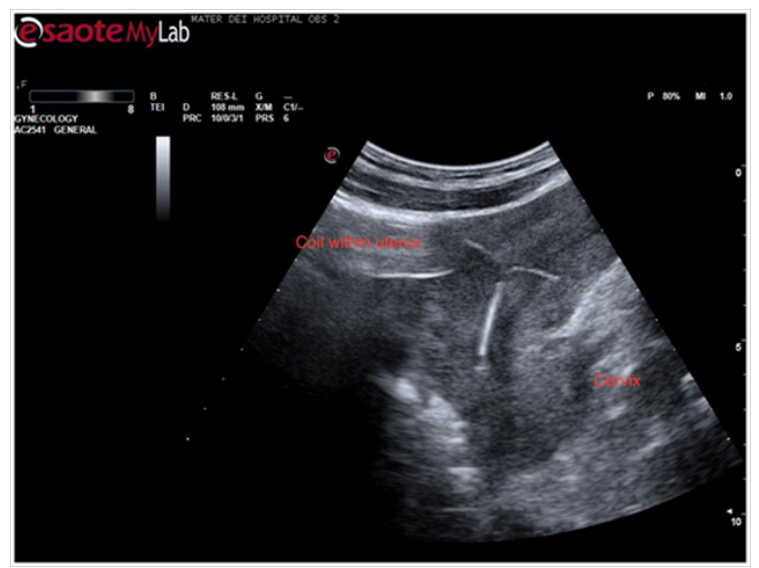

Figure 3 Sagittal view of the uterus with coil seen in its entire length within endometrial cavity.

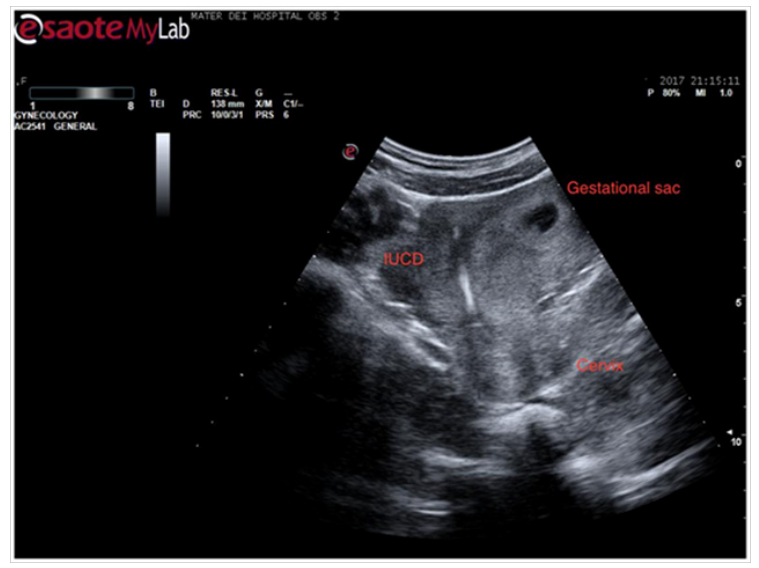

Figure 4 Sagittal view of the uterus showing both coil and gestation sac.
At this stage a decision was made to proceed to transvaginal scan in the hope of mapping the coils' location better. Once again verbal consent was obtained and after covering the patient with a modesty sheet the high-frequency endovaginal probe was gently introduced after covering with gel and a probe cover. The images obtained confirmed the findings of the abdominal scan (Figure 5). By rotating the probe anticlockwise the uterus could be visualised in its transverse diameter. This view is a good one for demonstrating separation of the endometrial stripe into 2 as the uterus is scanned from a caudal to a cephalic direction. On reaching the fundus 2 definite horns are usually visualized (Figure 6). In this case, a gestation sac was distending the left horn as demonstrated in Figure 7. The rest of the examination was normal with both adnexae visualised and appearing normal.

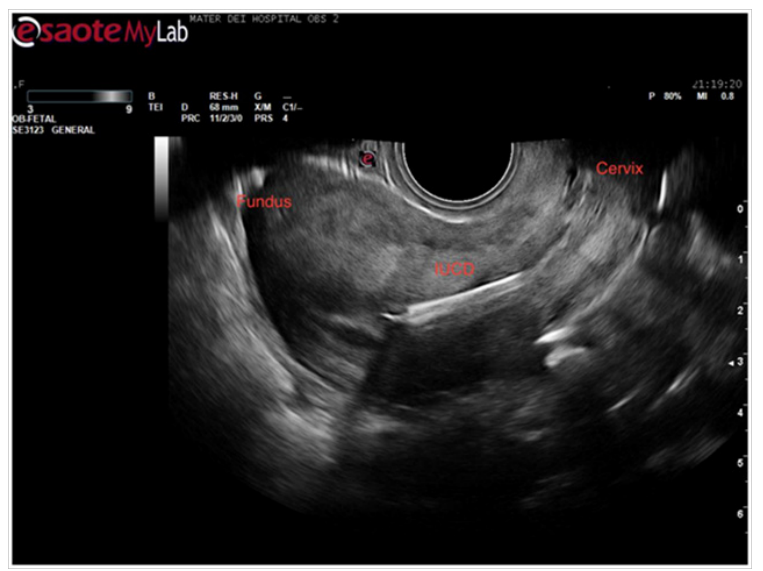

Figure 5 Sagittal view of uterus demonstrating right horn of endometrial cavity with echogenic coil within.

The findings were explained to the patient who was understandably upset. She was given time to ask questions and a follow-up appointment was given for the following day for a departmental scan and discussion regarding further management as per hospital protocol. The findings were confirmed the following day and the patient requested more time to evaluate her options. She refused to have the coil removed. She was unfortunately lost to follow up.

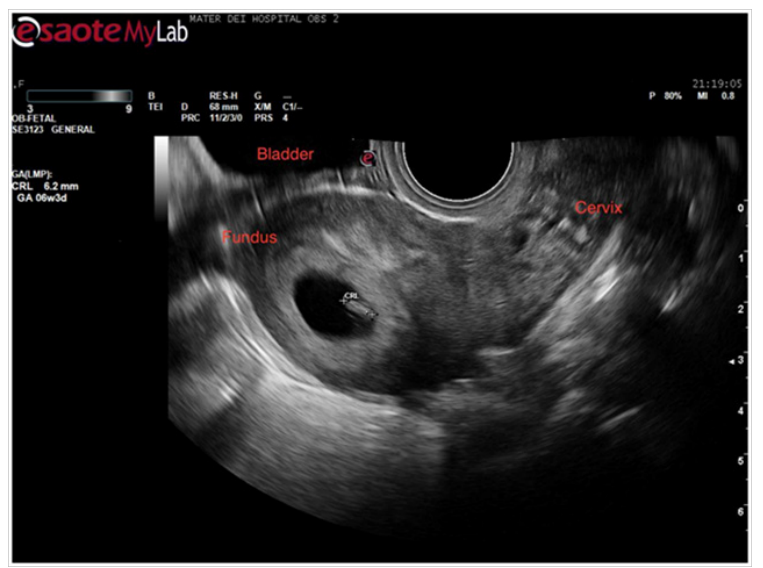

Figure 6 Sagittal view of uterus demonstrating intrauterine sac with fetal pole equivalent to 6 weeks and 3 days gestation.

\section{Discussion}

This case highlights the important role of ultrasound imaging both in the diagnosis of congenital abnormalities of the uterus as well as in patients using intrauterine contraceptive devices. Congenital malformations of the female genital tract are defined as deviations 
from normal anatomy resulting from embryological mal development of the Mullerian or paramesonephric ducts and they represent a rather common benign condition with a prevalence of $4-7 \% .^{7}$ These anomalies are associated with health and reproductive problems depending on the type and the degree of anatomical distortion. ${ }^{8}$ This implies that determining the exact nature and severity of the abnormality is crucial to the counselling and management of these patients. Several modalities have been compared for the diagnosis of such conditions. Studies quote a $90-92 \%$ accuracy in diagnosing congenital uterine anomalies for $2 \mathrm{D}$ ultrasound however its sensitivity is quite low. ${ }^{9}$ Combined with hysterosalpingogram it could prove to be a very effective screening tool as it is a widely available and noninvasive method. Combined hysteroscopy and laparoscopy allows for a direct visualisation of the external and internal contour of the uterus and was till recently considered to be the gold standard for diagnosis. ${ }^{10}$ In more recent years $3 \mathrm{D}$ ultrasound and MRI have also been increasingly adopted. The former is non invasive and has been reported to have a very high accuracy rate in diagnosis of congenital uterine anomalies. ${ }^{11}$ Most types of uterine anomalies can nowadays be confidently diagnosed using pelvic MRI. ${ }^{12}$ This is now considered the standard for imaging uterine anomalies as it provides high resolution images and in addition can help to evaluate urinary tract for concomitant anomalies. ${ }^{13}$ This particular case demonstrates how very often such anomalies are picked up incidentally. Such cases require a lengthy discussion with the patient which in this case proved difficult due to a language barrier. In ideal circumstances, an interpreter would have proved helpful to explain the ultrasound findings and its implications for the future.

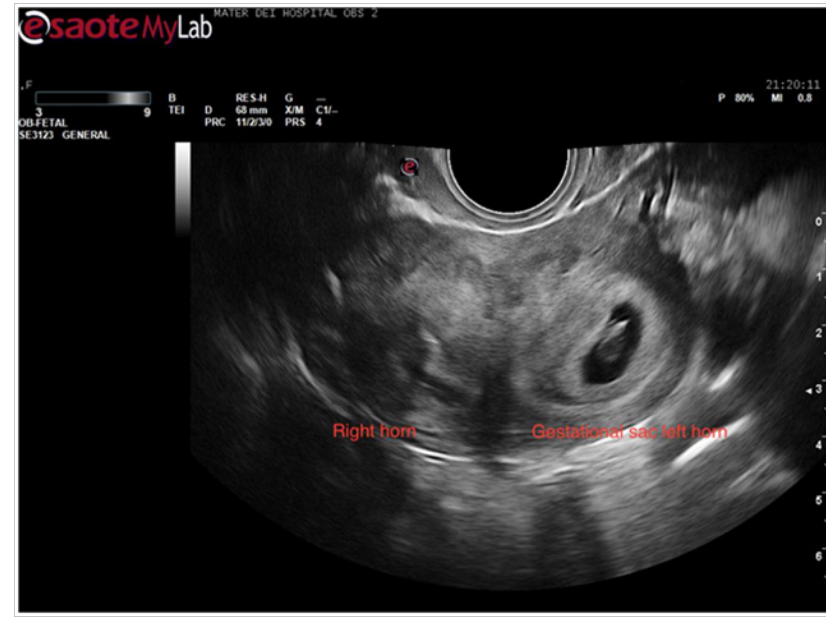

Figure 7 Transvaginal scan showing a transverse view of the uterine fundus with 2 separate horns.

Few cases of pregnancies in a bicornuate uterus with an IUD are reported in the literature and no studies as such have been carried out in terms of out-comes and live birth rates. In clinical practice, if major uterine anomalies are diagnosed they are considered to be a contraindication to IUD use. Very often the presence of the anomaly will not be known at the time of the IUD insertion as in this case. It does seem likely that the effectiveness of the IUD may be reduced among women with 2 uterine cavities. The risk of pregnancy with an IUD is reported to be highest in the first year of insertion. Very often sonography is used as the most common initial method of evaluation of IUD placement due to its cost effectiveness, lack of ionizing radiation and greater detail of pelvic anatomy. ${ }^{14}$ When a pregnancy is suspected an ultrasound is even more crucial to determine the location of gestation. Studies show that confirmed pregnancies with an IUD in situ are at risk for adverse pregnancy outcomes including spontaneous abortion, preterm delivery, septic abortion and chorioamnionitis. ${ }^{15}$ It seems that the risk of adverse pregnancy outcomes is lower if the IUD is removed compared with those patients who retain it. ${ }^{16}$

In this case, the patient did not wish to have the IUD removed at the first encounter which is understandable as she had still not come to terms with the pregnancy. Unfortunately, she was lost to follow-up as it would have been very interesting to follow the course of this pregnancy whichever option she ultimately chose to go for.

\section{Acknowledgments}

None.

\section{Conflicts of interest}

None.

\section{References}

1. American College of Obstetricians and Gynaecologists Committee on Gynecologic Practice, Long Acting Reversible Contraception Working Group. ACOG Committee Opinion no 450: Increasing use of contraceptive implants and intrauterine devices to reduce unintended pregnancy. Obstet Gynecol. 2009;114(6):1434-1438.

2. Goodwin T Murphy, Montoro Martin N, Muderspach Laila, et al. Management of Common Problems in Obstetrics and Gynaecology. (5th edn), John Wiley and Sons, USA. 2010;pp.494-496.

3. Junqueira BL, Allen LM, Spitzer RF, et al. Mullerian duct anomalies and mimics in children and adolescents: correlative intraoperative assessment with clinical imaging. Radiographics. 2009;29(4):10851103.

4. Troiano RN, Mccarthy SM. Mullerian duct anomalies: imaging and clinical issues. Radiology. 2004;233(1):19-34.

5. Nahum GG. Uterine anomalies. How common are they and what is their distribution among subtypes? J Reprod Med. 1998;43(10):877-887.

6. Rossing MA, Daling JR, Voigt LF, et al. Current use of an intrauterine device and risk of tubal pregnancy. Epidemiology. 1993;4(3):252-258.

7. Grimbizis GF, Campo R. Congenital malformations of the female genital tract: the need for a new classification system. Fertil Steril. 2010;94(2010):401-407.

8. Grimbizis GF, Camus M, Tarlatzis BC, et al. Clinical implications for uterine malformations and hysteroscopic treatment results. Hum Reprod Update. 2001;7(2):161-164.

9. Byrne J, Nussbaum-Blask A, Taylor WS, et al. Prevalence of Mullerian duct anomalies detected at ultrasound. Am J Med Genet. 2000;94(1):912.

10. Saravelos SH, Cocksedge KA, Li TC. Prevalence and diagnosis of congenital anomalies in women with reproductive failure: a critical appraisal. Hum Reprod Update. 2008;14(5):415-429.

11. Wu MH, Hsu CC, Huang KE. Detection of congenital Mullerian duct anomalies using three-dimensional ultrasound. J Cli Ultrasound. 1997;25(9):487-492.

12. Marcela L, Nothaft MA, Coelho F, et al. Müllerian duct anomalies: MR Imaging. Abdom Imaging. 2011;36(6):756-364.

13. Graupera B, Pascual MA, Hereter L, et al. Accuracy of threedimensional ultrasound compared with magnetic resonance imaging in diagnosis of Müllerian duct anomalies using ESHRE-ESGE consensus on the classification of congenital anomalies of the female genital tract. Ultrasound Obstet Gynecol. 2015;46(5):616-622. 
14. Peri N, Graham D, Levine D. Imaging of intrauterine contraceptive devices. J Ultrasound Med. 2007;26(10):1389-1401.

15. Brahmi D, Steenland MW, Renner RM, et al. Pregnancy outcomes with an IUD in situ: a systematic review. Contraception. 2012;85(2):131139.
16. Ozgu-Erdinc AS, Tasdemir UG, Uygur D, et al. Outcome of intrauterine pregnancies with intrauterine device in place and effects of device location on prognosis. Contraception. 2014;89(5):426-430. 